\title{
Safety and efficacy of biodegradable stents in octogenarian patients with esophageal achalasia
}

\section{다)(요 $\odot$}

\author{
Authors \\ Murcio-Pérez ${ }^{1,2}$ \\ Institutions \\ 1 Instituto Mexicano del Seguro Social - Digestive \\ Endoscopy, Ciudad de Mexico, Mexico \\ 2 Hospital de Especialidades Centro Medico Nacional \\ Siglo, Mexico City, Mexico
}

Oscar Hernandez-Mondragon ${ }^{1}$, Luis Garcia Contreras ${ }^{1}$, Omar Michel Pineda ${ }^{1}$, Geraro Blanco-Velasco ${ }^{1}$, Enrique

submitted 23.11.2020

accepted after revision 5.1.2021

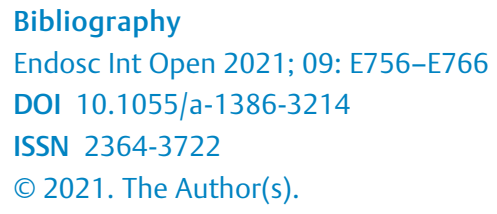

\section{ABSTRACT}

Backgrounds and study aims Treatment of octogenarian patients with achalasia with conventional treatments is ef- fective but with compromised safety. Biodegradable stents (BS) are promising. We aimed to evaluate their safety, efficacy and clinical outcomes at early, mid and long-term in this population.

Patients and methods Naïve or previously-treated achalasic octogenarian patients underwent to BS placement (BSP) between December, 2010 and November, 2011, and were followed-up for 9-years. A strict follow-up was performed.

Results Thirty-two patients were included, (17 men [53.1\%]; median age 82 years [78-92]). BSP was performed in all patients. At 9y, 18/32 (56.2\%) completed protocol. Mean BSP time was $37.5 \pm 12.1 \mathrm{~min}$ and $34.4 \%$ presented thoracic pain. At $1 \mathrm{~m}$, six BS were migrated (18.7\%), requiring a second BSP fixed with hemoclips. At $3 \mathrm{~m}$, twenty-three (72.8\%) completed degradation process. At $6 \mathrm{~m}$, eighteen (56.2\%) presented clinical dysphagia, of whom 5/32 (15.6\%) presented stenotic-tissue hyperplasia, responding to balloon dilation in all cases. Pre-BSP Eckardt, Timed barium esophagram and integrated relaxation pressure improved post-BSP $6 \mathrm{~m}$ values ( 9 vs $2, \mathrm{p}=0.001 ;<50 \%=$ $93.8 \%$ vs $>80 \%=81.5 \%, p=0.003$ and $18.8 \pm 3.2$ vs $11.1 \pm$ $2.6 \mathrm{mmHg}, \mathrm{p}=0.001$, respectively), and there were no significant changes up to $9 y$ post-BSP. Esophagitis grade A or $B$ was presented between $4.7 \%$ to $11.2 \%$ and controlled with PPI. After 9 years we had clinical success rates of $94.4 \%, 72 \%$, and $65.4 \%$ for time point evaluation, per protocol and intention to treat analysis, respectively.

Conclusions BSP represents a feasible alternative option in octogenarian patients with achalasia who are high risk with other treatments, presenting acceptable early, mid-, and long-term outcomes.

\section{Introduction}

Esophageal achalasia is a primary esophageal motor disorder characterized by aperistalsis and failure in lower esophageal sphincter (LES) relaxation [1]. Treatment aim is to decrease LES pressure and integrated relaxation pressure (IRP), in order to improve symptoms and quality of life [2,3]. Laparoscopic Heller Myotomy (LHM) and Peroral endoscopic myotomy
(POEM) are considered the gold standard of treatment [4-6]; however, safety is compromised in some populations, such as in elderly patients (adverse events of $2 \%$ to $11.5 \%$ in LHM and $5 \%$ to $7.8 \%$ for POEM [7-9]), representing high-risk procedures for them. Alternative treatments include pneumatic dilation (PD) (73\%-88\% early and mid-term efficacy, but a median of $1.9 \%$ perforation rate [4-10]) and botulinum toxin injection (BTI) (initial $87 \%$ to $92 \%$, but $22 \%$ to $31 \%$ mid-term efficacy 
$[8,11])$. Fully or partially covered self-expandable metal stents (SEMS) have been used with variable clinical remission rates ( $88 \%$ to $100 \%$ at early-term and $49 \%$ to $91 \%$ at long-term $[12,13]$ ), but with concerning adverse events (AEs) (thoracic pain $35 \%$ to $44 \%$, reflux symptoms $28 \%$ to $36 \%$, migration $8.5 \%$ to $18 \%$, and bleeding $8 \%$ to $12 \%$ [14-16]).

Biodegradable stents have appeared as a promising alternative for different gastrointestinal diseases, including benign esophageal strictures (BES) $[17,18]$. Their main advantage is the ability of exert a continuous radial force that last up to 6 weeks before degradation process begins, avoiding the need for endoscopic removal [19]. In peptic BES, promising results have been observed in $65 \%$ to $87.5 \%, 45 \%$ to $60 \%$ and $25 \%$ at early, mid- and long-term evaluations, respectively [20], with AEs that include migration ( $8 \%-20 \%)$, thoracic pain (10\%-57\%) and tissue hyperplasia (5\%-60\%) [21,22], representing a good alternative of treatment in achalasic patients, especially in high-risk groups such as octogenarians. Therefore, we aimed to investigate the safety and efficacy of BS in a group of octogenarian patients with achalasia.

\section{Patients and methods}

\section{Study design and ethical considerations}

This was a prospective study performed between December, 2010 and October, 2020 in a tertiary-care center in Mexico City, Mexico. We included all octogenarian patients with naïve or previously-treated achalasia at any stage who were at high risk for surgery (e.g. patients with severe cardiopulmonary conditions, significant coagulation disorders, etc.), but without contraindication to an upper endoscopy (UE) or those who didn't accept other treatment modalities such as POEM or PD. Patients who were unable to receive general anesthesia, those with pseudoachalasia, esophagogastric tumors, peptic strictures, or suspicion of gastrointestinal malignancy were excluded. All BS were placed between December, 2010 and November, 2011, and then completed protocol up to October, 2020.

This protocol was approved on sept 22, 2010, by the Local Ethics Committee (R-2010-3601-096; trial registration number: 2010-CMN112). Informed consent was obtained from all patients.

\section{Patients}

Diagnosis of achalasia was based on clinical, radiological, endoscopic and manometric characteristics [4]. All patients underwent UE, chest computed tomography, Chagas testing, highresolution manometry (HRM) using Chicago's classification for achalasia subtypes [23,24], and timed barium esophagram (TBE) (at 1, 2, and 5 minutes). Clinical evaluation was done using the Eckardt scale [25].

\section{Biodegradable stent placement}

First, the esophagus was cleaned before BSP. Esophageal classification was evaluated according to Rezende's classification [26]. All procedures were performed under deep-sedation anesthesia and fluoroscopy guidance. A 9.8-mm outer diameter with a 2.8-mm working channel endoscope was used (EG-
450WR5 or EG590WR; Fujinon, Tokyo, Japan). After endoscopic revision and esophagogastric junction (EGJ) level documentation, $1 \mathrm{~mL}$ of non-ionic contrast agent at $50 \%$ (Omnipaque, GE Healthcare, Ireland Limited, Cork, Ireland) was injected at submucosal level $1 \mathrm{~cm}$ above EG]. Then, a metallic $200 \mathrm{~cm}$ long Savary-Gilliard wire-guide (Cook Medical, Bloomington, Indiana, United States) was introduced and placed on gastric antrum. Finally, a polydioxanone biodegradable stent of $25 \mathrm{~mm} \times 60 \mathrm{~mm}$ (Ella-CS, Hradec Kralove, Czech Republic) was placed under fluoroscopic guidance throughout the guide and deployed, assuring that the middle part of the stent was placed over the submucosal marker. Final endoscopic review was performed and confirmation of adequate BSP documented ( $\mathbf{F i g} \mathbf{1}$ ).

\section{Follow-up}

After the procedure, patients were hospitalized for detection of potential adverse events $(A E)$, then liquid diet was initiated and continued for 2 days, and progressed to soft by 3 days and then normal diet. Proton pump inhibitors (PPI) were administered initially for 3 months to prevent reflux and avoid premature stent degradation, and then accordingly to results of reflux assessment. Patients underwent clinical (dysphagia evaluated according to Dakkak and Bennett score) [27], and radiological assessment at 1 and 2 months. Then, UE, TBE, HRM, Eckardt and esophageal 24-hour $\mathrm{pH}$ monitoring were performed at 3 and 6 months, and then annually for up to 9 years. If stenotic tissular hyperplasia (STH) was found on UE, patients underwent balloon dilation program up to $15 \mathrm{~mm}$ with CRE balloon (Boston Scientific, Natick, Massachusetts, USA). If migration of stent was presented, a second BS of the same characteristics was inserted and fixed with hemoclips to avoid migration (Boston Scientific, Natick, Massachusetts, United States).

Clinical success (CS) was defined when after 6 months of BSP, we observed an Eckard score $<3, \mathrm{TBE} \geq 80 \%$ at 5 minutes, and integrated relaxation pressure (IRP) $<15 \mathrm{mmHg}$; failure when Eckardt $\geq 3$, TBE $<50 \%$ and $I R P \geq 15 \mathrm{mmHg}$; partial response (PR), when patients had an Eckardt $\geq 3$ and IRP $\geq 15 \mathrm{mmHg}$ or delayed TBE (between $50 \%$ to $80 \%$ or $<50 \%$ ). Finally, IRP or TBE failure criteria alone were not considered as patients with PR. When PR was found after two consecutive follow-ups, they were considered as failures and underwent PD, as rescue therapy with Rigiflex balloon (Microvasive, Watertown, Massachusetts). In cases with failure criteria, if a second follow-up showed failure criteria again or PR, they also underwent the PD program. If esophagitis on UE or 24-hour $\mathrm{pH}$ monitoring were documented, PPI was administered indefinitely. Adverse events were graded according to the American Society for Gastrointestinal Endoscopy Lexicon [28].

\section{Statistical analyses}

The sample size was calculated based on the formula for difference of proportions for paired measurements (clinical improvement before and after BSP). According to previous studies [12$16]$, there is a mean $80 \%$ clinical improvement after procedure. Thus, in spite of no previous BSP studies in achalasic patients, we hypothesized an improvement of at least $80 \%$, assuming a $20 \%$ dropout rate with a significance alpha level of 0.05 (Type I 

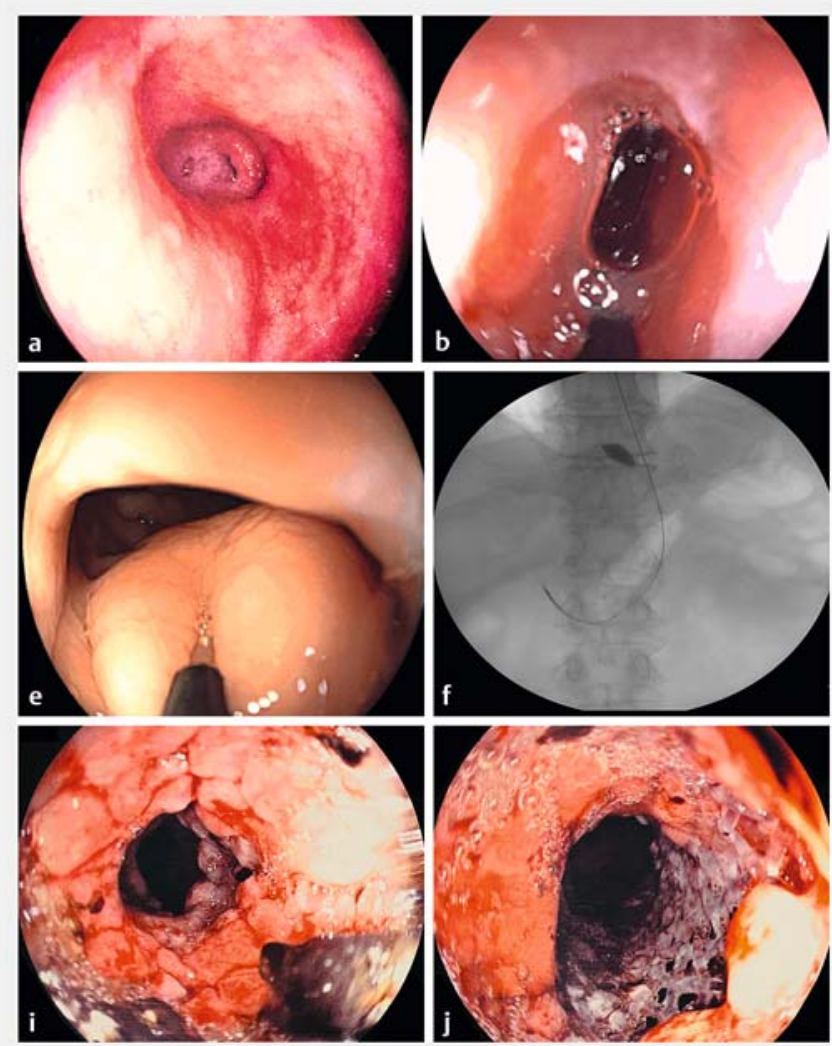
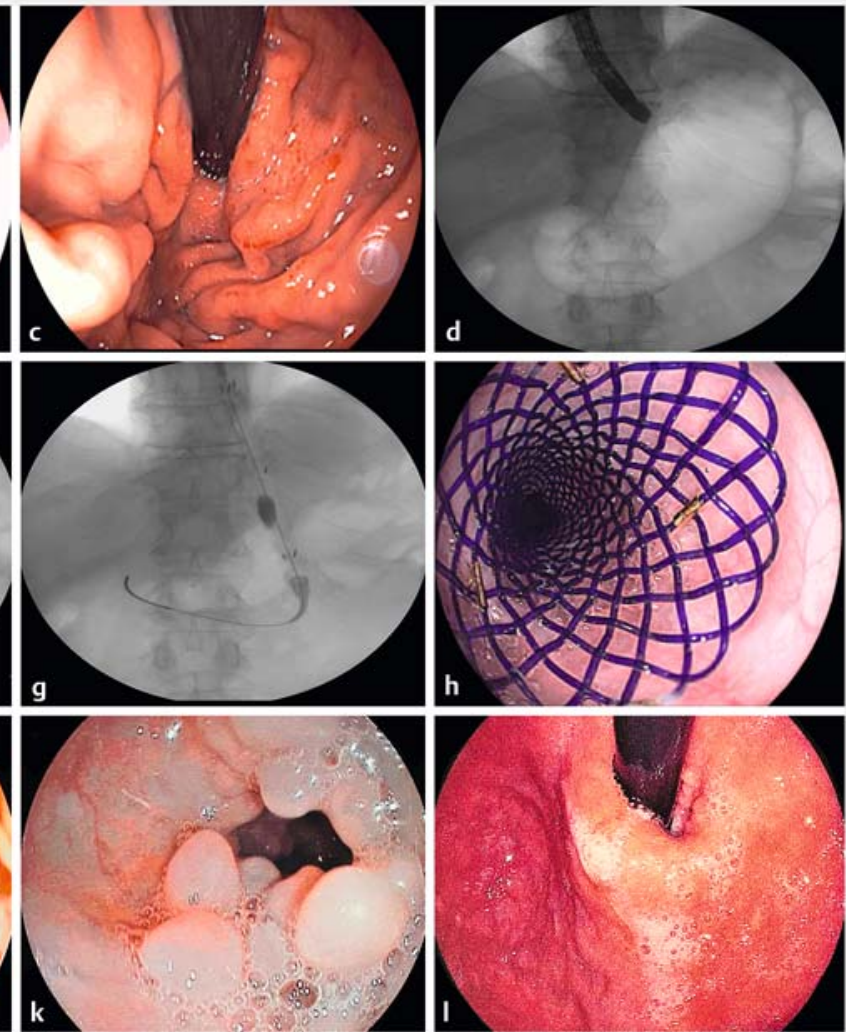

- Fig. 1 Biodegradable stent placement technique and changes at 3 and 7 years after. a Octogenarian patient with Type I achalasia, showing a grade III dilated esophagus. b EGJ without mucosal abnormalities. c Retroflexion view. d Radiological view of EGJ position. e Injection of $1 \mathrm{~mL}$ of non-ionic contrast agent at $50 \% 1 \mathrm{~cm}$ above EGJ. f Fluoroscopic view of submucosal injection of contrast, and Savary guidewire placement. g Biodegradable stent placement with fluoroscopic guidance. $\mathbf{h}$ Endoscopic view of biodegradable stent in adequate position. i Partial degradation process after 3 months of BSP. $\mathbf{j}$ Non-stenotic tissular hyperplastic reaction at EG] level. $\mathbf{k}$ Same patient, showing non-stenotic mucosal changes at 7 years after BSP. I Retroflexion view showing a slightly opened EGJ with hyperplasic mucosal changes of biodegradable stent.

error of $5 \%$ ) and a beta of 0.20 (Type II error of $20 \%$ ). Using an online statistically-validated program of sample size calculation (Epilnfo, United States), we calculated a sample of 20 patients.

The clinical characteristics of patients, procedures and outcomes are documented as mean with standard deviation (SD) or medians with interquartile ranges (IQR) for quantitative variables, according to their distribution. Qualitative data are expressed as frequencies and percentages. Evaluation of BSP outcomes were performed using Friedman, Wilcoxon, Student's t-test and ANOVA tests for quantitative data, and linearby-linear association test for qualitative variables. CS was evaluated according to per protocol (PP) and intention to treat (ITT) analysis and time point evaluation (TPE). $P<0.05$ was considered statistically significant. SPSS 23.0 for Mac (IBM) was used for statistical analysis.

\section{Results}

\section{Baseline characteristics}

Of 37 octogenarian patients with achalasia, five were excluded. Thirty-two were included for BSP that was performed successfully in all cases between December, 2010 and November, 2011
(17 men [53.1\%]; median age 82 years [78-92]). After 9 years, 18 of 32 patients (56.2\%) completed 9-year follow-up ( $\triangleright$ Fig. 2).

The median body mass index (BMI) was 19.2 (14.2-24.1). The most common achalasia subtype was Type I in $62.5 \%$. Grade II, III and IV were found in $34.4 \%, 25 \%$, and $15.6 \%$, respectively. Twenty-one (65.6\%), were previously treated (PD in $28.1 \%$ ). The median Eckardt pre-BSP was 9 (6-12), and $93.8 \%$ of the patients had a pre-BSP TBE $<50 \%$ ( $>$ Table 1 ).

\section{Biodegradable stent placement (early outcomes)}

The mean BSP time was $37.5 \pm 12.1$ minutes. Chest pain was present in $34.4 \%$ (controlled with nonsteroidal anti-inflammatory drugs [NSAIDs]), followed by transprocedural self-limited mild bleeding (12.5\%). The median length of stay was 1 day (1-2), diet was progressed successfully.

At 1 month, we had six migrations, requiring a second stent placement, fixed with a median of three hemoclips (2-4). At 2 months all stents were in place. At 3 months, complete and partial degradation of the stent were present in $23(72.8 \%)$ and nine $(27.2 \%)$, respectively. 
- No migration

- No clinical stenosis

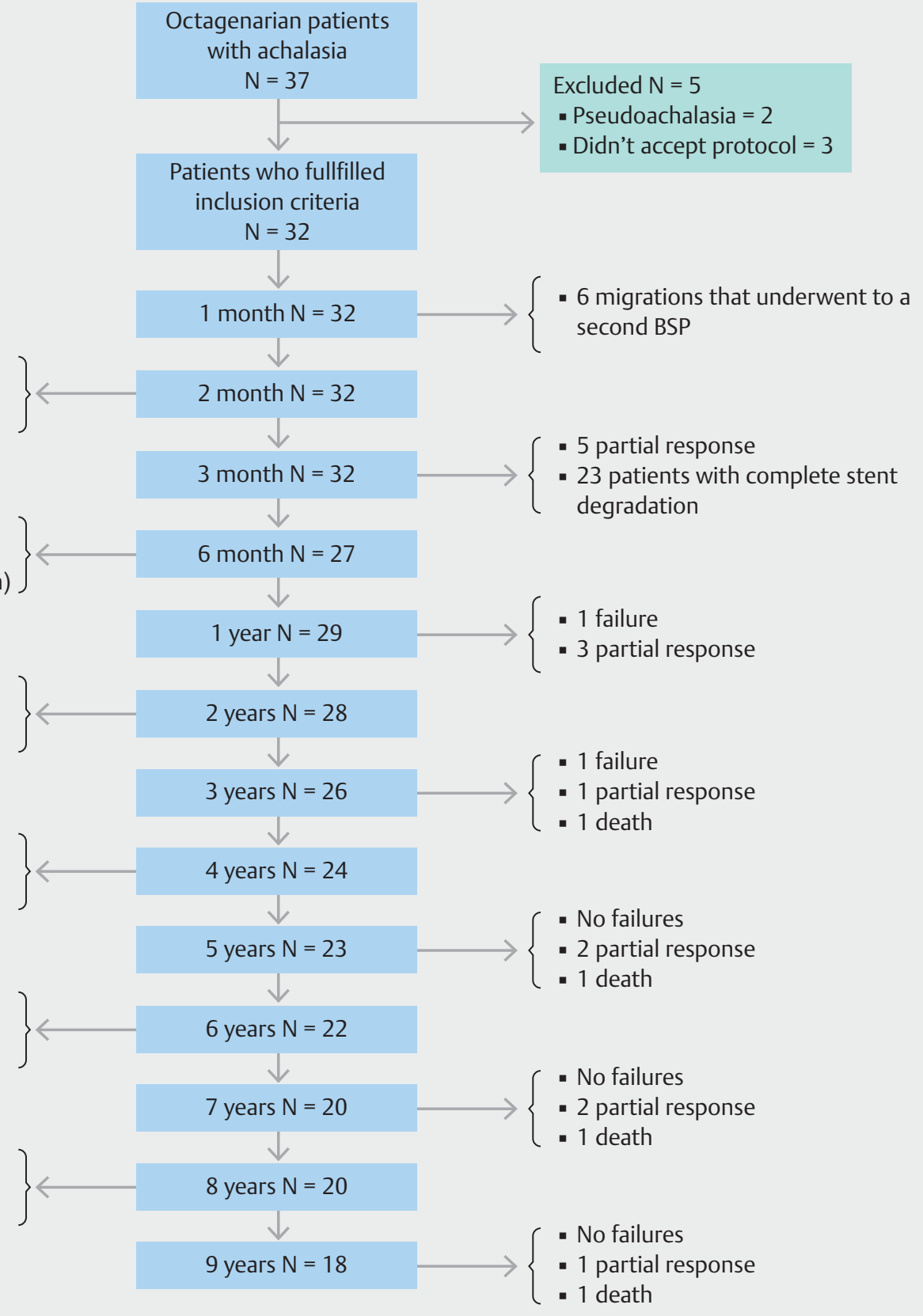

- Fig. 2 Flowchart of the cohort of octogenarian patients with achalasia.

\section{Endoscopic stenosis after BSP}

No dysphagia was present before 3 months; however, at 3 months post-BSP, 12 of 32 (37.5\%) and three of 32 (9.3\%), presented dysphagia to some solid and semi-solid food, respectively. For UE, $50 \%$ presented with an opened esophagogastric junction (OEGJ), and $50 \%$ non-stenotic tissular hyperplasia (NSTH).

At 6 months, we had some degree of dysphagia in 18 of 32 (56.2\%), in 10 of whom (31.3\%) it was to solid food, not requiring endoscopic treatment. However, it was clinically significant in eight of 32 patients (25\%), in five of whom (15.6\%) it was to semi-solid food $(\mathrm{NSTH}=2 / \mathrm{STH}=3)$, and in three $(9.3 \%)$, to liquids (NSTH=1/STH=2). All STH (5/32 [15.6\%]), required CRE balloon dilation up to $15 \mathrm{~mm}$, requiring one, two, and three to five sessions in one, two, and two patients, respectively ( $\bullet$ Table 2).

\section{Biodegradable stent placement (early, mid-, and long-term outcomes)}

The median pre-BSP Eckardt score was 9 (6-12), and decreased to $4(2-6)$ after 1 month $(P=0.001)$, and maintained up to 9 years (2 [1-3]; $P=0.14)$. Dakkak score didn't show changes between pre-BSP and post-BSP 1-month and 2-month values; 
- Table 1 Characteristics of patients and procedures.

\begin{tabular}{|c|c|}
\hline & $\mathrm{N}=32$ \\
\hline Age, median (IQR), years & $82(78-92)$ \\
\hline Gender, n (\%), male & $17(53.1 \%)$ \\
\hline BMI, median (IQR), score & $19.2(14.2-24.1)$ \\
\hline $\begin{array}{l}\text { Time before achalasia diagnosis, median (IQR), } \\
\text { months }\end{array}$ & $210(22-601)$ \\
\hline \multicolumn{2}{|l|}{ Previous treatments, (\%) } \\
\hline - Treatment naïve & $11(34.4 \%)$ \\
\hline - Previously treated & $21(65.6 \%)$ \\
\hline - Post-LHM & $7(21.9 \%)$ \\
\hline - Botulinum toxin injection & $5(15.6 \%)$ \\
\hline - Pneumatic dilation & $9(28.1 \%)$ \\
\hline \multicolumn{2}{|l|}{ Achalasia subtype, n (\%) } \\
\hline - Type I & $20(62.5 \%)$ \\
\hline - Type II & $11(34.4 \%)$ \\
\hline - Type III & $1(3.1 \%)$ \\
\hline \multicolumn{2}{|l|}{ Type of esophagus, n (\%) } \\
\hline - Normal & $2(6.3 \%)$ \\
\hline - Grade I & $6(18.8 \%)$ \\
\hline - Grade II & $11(34.4 \%)$ \\
\hline - Grade III & $8(25 \%)$ \\
\hline - Grade IV & $5(15.6 \%)$ \\
\hline Eckardt pre-BSP, median (IQR), points & $9(6-12)$ \\
\hline IRP pre BSP, mean (SD), mmHg & $18.8 \pm 3.2$ \\
\hline \multicolumn{2}{|l|}{ TBE pre BSP, n (\%) } \\
\hline . $<50 \%$ & $30(93.8 \%)$ \\
\hline - $50 \%-80 \%$ & $2(6.2 \%)$ \\
\hline " $>80 \%$ & $0(0 \%)$ \\
\hline BSP duration, mean (SD), min & $37.5 \pm 12.1$ \\
\hline \multicolumn{2}{|l|}{ Adverse events, n (\%) } \\
\hline - Chest pain & $11(34.4 \%)$ \\
\hline - Bleeding & $4(12.5 \%)$ \\
\hline - None & $17(53.1 \%)$ \\
\hline \multicolumn{2}{|c|}{$\begin{array}{l}\text { SD, standard deviation; IQR, interquartile range; BMI, body mass index; } \\
\text { LHM, laparoscopic heller myotomy; IRP, integrated relaxation pressure; } \\
\text { TBE, timed barium esophagram; BSP, biodegradable stent placement. }\end{array}$} \\
\hline
\end{tabular}

however, they improved at 3 months (pre-BSP $=4$ [2-4] vs postBSP 3 months $=10-2 ; P=0.001)$, and values were maintained up to 9 years (post-BSP 3 months $=10-2$ vs post-BSP 9 years $=$ 2 0-2; $P=0.09$ ).

The IRP pressure improved from pre-BSP $=18.8 \pm 3.2 \mathrm{mmHg}$ to post-BSP 3 months $=12 \pm 2.6 \mathrm{mmHg}(P=0.001)$; and main- tained up to 9 years. DeMeester score after BSP was stable from 3 months to 9 years (post-BSP 3 months $=11.8 \pm 5.0$ vs post-BSP 9 years $=10.8 \pm 2.1 ; P=0.12$ ).

Pre-BSP TBE was $<50 \%$ in $93.8 \%$ and improved to post-BSP TBE $>80 \%$ in $71.9 \%, 81.5 \%, 93 \%$, and $80.8 \%$ for 3 months, 6 months, 1 years and 2 years, respectively $(P=0.003)$. After 3 years post-BSP TBE was maintained up to 9 years $(P=0.11)$, and up to $61.1 \%$ of our patients presented a TBE $>80 \%$ after 9 years of BSP ( $\mathbf{F i g . 3}$ ).

Pre-BSP UE showed a closed esophagogastric junction (CEG]) in all cases, which improved to post-BSP at 3 months (NSTH= $18 / 32[56.3 \%]$ and $\mathrm{OEG}=14 / 32[43.8 \%] ; \mathrm{P}=0.03)$. At postBSP 6 months, five of 32 (15.6\%) changed from NSTH to STH $(P=0.01)$, and were temporarily separated from the cohort ( $n$ $=27$ ), once the dilation program ended, they were included at 1-year follow-up. At post-BSP 1 year, 72.4\% presented OEGJ, 20.7\% NSTH, 10.3\% LGE, and 6.9\% CEGJ. Similar values were found at 9 years without statistically significant differences ( $>$ Table 3 ).

Regarding TPE assessment, all patients were failed before BSP and improved by post-BSP 6 months $(C S=22$ [81.5\%], PR = $2[7.4 \%]$ and failure $=3[11.1 \%] ; P=0.001)$, and improved at post-BSP 1 year $(C S=25[86.2 \%], P R=3[10.3 \%]$ and failure $=1$ [3.5\%]; $P=0.003)$. No changes were found up to post-BSP 9 years $(C S=17$ [94.4\%], $P R=1$ [5.6\%] and failure $=0$ [0\%]; $P=$ $0.19)$. In the PP analysis, we had a final post-BSP 9 years: $C S=$ 17 of $26(65.4 \%)$; PR = one of $26(3.9 \%)$ and failure=eight of $26(30.7 \%)$, and in the ITT, we had CS=23 of $32(72 \%)$; PR = one of 32 (3\%) and failure=eight of 32 (25\%) (> Fig. 4). We had six deaths (18.8\%) (not related to BSP and all were successful before they died). Finally, we performed a bivariate analysis of success and failure patients at 9 years after BSP, including clinically-relevant variables; however, we observed no differences between groups.

\section{Discussion}

In this paper, we evaluated the feasibility, safety and efficacy of BSP in a group of octogenarian patients with achalasia, at early, mid and long-term.

POEM and LHM represent the cornerstone of treatment in young patients. POEM has a clinical success in octogenarians of $>90 \%$, but AE ranging from $5-7.8 \%$, most of them mild, but up to $10.7 \%$ could be severe [7,29-31]. LHM has been associated with perioperative complications (bleeding [5-18\%], perforation [2\%-5\%], postsurgical infection [3\%-6\%]) [8,9,32, 33]. PD has a median perforation rate of $1.9 \%[4,8]$, and BTI has a temporary effect (mid-term efficacy $22 \%-31 \%$ ) [11]; moreover, if a more definite treatment is offered, submucosal fibrosis and mucosal injury rate are increased (4\%-12\%) [2, 4, 8]. Other options include fully or partially covered SEMS, with variable results (49\%-91\% long-term efficacy and 8\%-45\% AE) [14-16].

Therefore, we considered the use of BSP, based on two reasons: first, because of its safety and efficacy observed in other gastrointestinal diseases, such as BES and small bowel strictures [17]. Second, BSP is performed in a conventional fluoroscopy 
- Table 2 Endoscopic stenosis after biodegradable stent placement.

\begin{tabular}{|c|c|c|c|c|c|c|c|c|c|c|c|}
\hline \multirow[b]{2}{*}{ Clinical dysphagia } & \multirow[t]{2}{*}{$\begin{array}{l}3 \text { months } \\
\mathrm{N}=32\end{array}$} & \multicolumn{3}{|c|}{$\begin{array}{l}\text { Endoscopic evaluation } \\
\text { at } 3 \text { months } \\
\mathrm{n}=32\end{array}$} & \multirow[t]{2}{*}{$\begin{array}{l}6 \text { months } \\
\mathrm{N}=32\end{array}$} & \multicolumn{3}{|c|}{$\begin{array}{l}\text { Endoscopic evaluation at } \\
6 \text { months } \\
n=32\end{array}$} & \multicolumn{3}{|c|}{$\begin{array}{l}\text { Dilation endoscopic } \\
\text { sessions } \\
n=5\end{array}$} \\
\hline & & NSTH & STH & OEG] & & NSTH & STH & OEG] & 1 & 2 & $3-5$ \\
\hline None $(0)$ & $17(53.2 \%)$ & $4(23.5 \%)$ & $0(0 \%)$ & $13(76.5 \%)$ & $14(43.8 \%)$ & $4(28.6 \%)$ & $0(0 \%)$ & $10(71.4 \%)$ & $0(0 \%)$ & $0(0 \%)$ & $0(0 \%)$ \\
\hline To some solid food (1) & $12(37.5 \%)$ & $9(75 \%)$ & $0(0 \%)$ & $3(25 \%)$ & $10(31.3 \%)$ & $6(60 \%)$ & $0(0 \%)$ & $4(40 \%)$ & $0(0 \%)$ & $0(0 \%)$ & $0(0 \%)$ \\
\hline To semisolid food (2) & $3(9.3 \%)$ & $3(100 \%)$ & $0(0 \%)$ & $0(0 \%)$ & $5(15.6 \%)$ & $2(40 \%)$ & $3(60 \%)$ & $0(0 \%)$ & $1(33 \%)$ & $2(66 \%)$ & $0(0 \%)$ \\
\hline To liquids (3) & $0(0 \%)$ & $0(0 \%)$ & $0(0 \%)$ & $0(0 \%)$ & $3(9.3 \%)$ & $1(33 \%)$ & $2(66 \%)$ & $0(0 \%)$ & $0(0 \%)$ & $0(0 \%)$ & $2(100 \%)$ \\
\hline Complete dysphagia (4) & $0(0 \%)$ & $0(0 \%)$ & $0(0 \%)$ & $0(0 \%)$ & $0(0 \%)$ & $0(0 \%)$ & $0(0 \%)$ & $0(0 \%)$ & $0(0 \%)$ & $0(0 \%)$ & $0(0 \%)$ \\
\hline
\end{tabular}
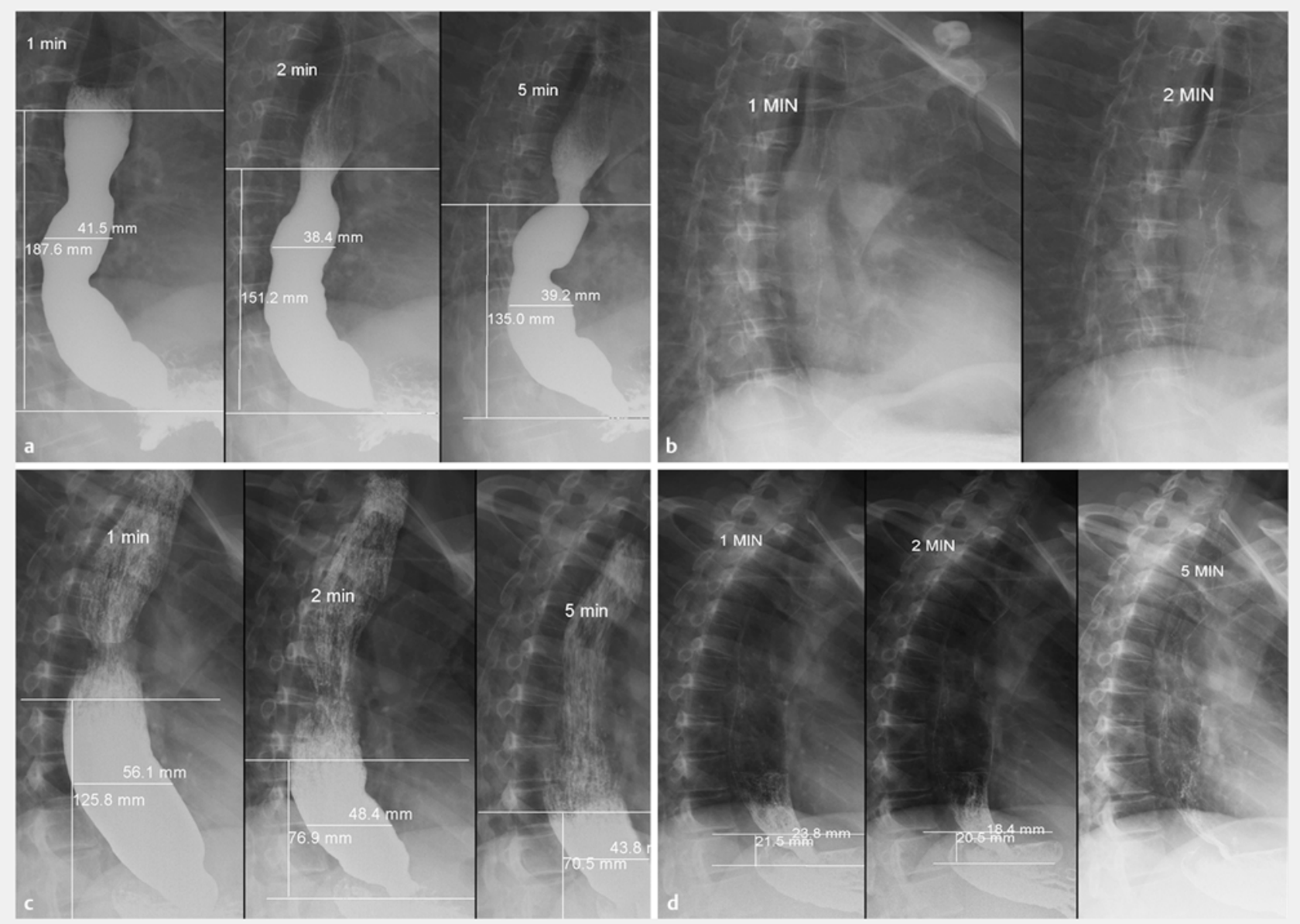

- Fig. 3 Timed barium esophagram emptying before and after BSP. a Emptying $<50 \%$ at 5 minutes before BSP was observed in a patient with sigmoid-type achalasia. b Emptying $>80 \%$ inclusive at 2 minutes was observed at 1 year after BSP. c Octogenarian patient with grade II achalasia showing emptying $<50 \%$ at 5 minutes. d More than $80 \%$ of emptying at 5 minutes was observed after 7 years after BSP.

and endoscopy suite, only deep sedation is needed, there is easy placement, and no removal is needed [18,20-22], representing a promising alternative in octogenarian patients with a high-risk nature (comorbidity, esophageal mucosal fragility, and anatomic esophageal changes due to aging) $[1,8,10,17]$. Diameter use was based on previous studies with SEMS. Cheng et al [12] compared the efficacy of a temporary use of 3 differ- ent diameter SEMS $(20,25$, and $25 \mathrm{~mm})$, in a prospective comparative study with a long-term follow-up (10 years); they concluded that $30 \mathrm{~mm}$ was superior and suggested that wider stents could have better outcomes. Therefore, we used the widest BS available ( $25 \mathrm{~mm})$.

Our octogenarian group had demographic characteristics similar to other authors that have studied and treated octogen- 


\begin{tabular}{|c|c|c|c|c|c|c|c|c|c|c|c|c|c|c|c|c|}
\hline & a & 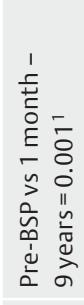 & 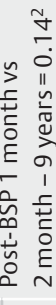 & & & & 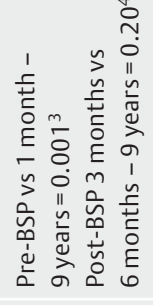 & & 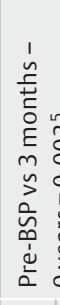 & 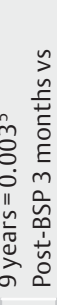 & 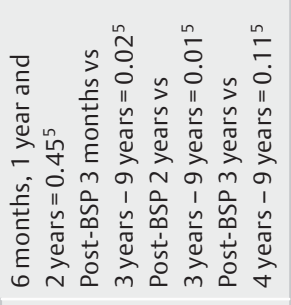 & 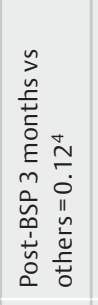 & 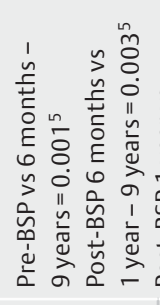 & 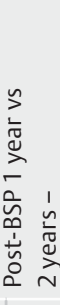 & 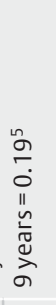 & \\
\hline & 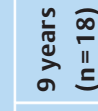 & 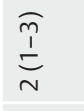 & 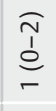 & $\begin{array}{c}\bar{c} \\
e \\
- \\
\end{array}$ & $\widehat{\widehat{o}}$ & $\widehat{o}$ & 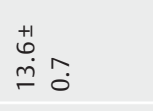 & & ồ & $\begin{array}{l}a \\
\infty \\
\infty \\
\stackrel{n}{n}\end{array}$ & $=\overline{\overline{\bar{\theta}}}$ & $\begin{array}{l}+1 \\
\stackrel{\infty}{\infty} \\
\stackrel{\sim}{-} \bar{i}\end{array}$ & & อิ & 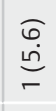 & 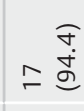 \\
\hline & 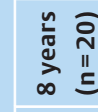 & 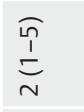 & $\begin{array}{c}\widehat{T} \\
d \\
c\end{array}$ & $\begin{array}{l}\widehat{T} \\
\mathfrak{d} \\
\underline{-}\end{array}$ & $\begin{array}{l}\overline{1} \\
1 \\
0 \\
0\end{array}$ & $\frac{\widehat{o}}{0}$ & $\stackrel{+1}{\stackrel{+}{m}} \stackrel{\sim}{\sim}$ & & $\stackrel{\sqrt{n}}{r}$ & $\underset{\infty}{\stackrel{o}{+}}$ & 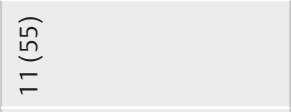 & $\begin{array}{l}\stackrel{+}{m} \stackrel{0}{\sim} \\
\sigma_{0}\end{array}$ & & $\stackrel{\pi}{\sqrt{n}}$ & $\underset{-}{\widehat{\omega}}$ & 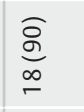 \\
\hline & 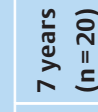 & 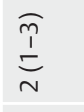 & $\begin{array}{c}\widehat{\hat{t}} \\
\stackrel{d}{\sigma}\end{array}$ & $\begin{array}{c}\bar{I} \\
\bar{c} \\
- \\
\end{array}$ & â & ô & $\stackrel{+1}{\stackrel{+}{\sim} \stackrel{\leftrightarrow}{\sim}}$ & & ồ & $\begin{array}{c}\widehat{0} \\
0 \\
0\end{array}$ & 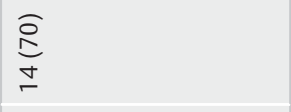 & $\begin{array}{l}\stackrel{+1}{\sim} \underset{\sim}{\sim} \\
\sim\end{array}$ & & ô & 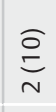 & 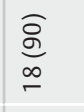 \\
\hline & 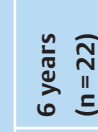 & $\underset{\substack{T \\
N}}{\stackrel{T}{n}}$ & 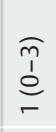 & $\begin{array}{c}\widehat{T} \\
\mathfrak{d} \\
- \\
\end{array}$ & $\begin{array}{l}\bar{I} \\
e \\
0\end{array}$ & 六 & 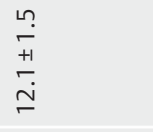 & & 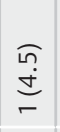 & $\begin{array}{c}\sigma \\
\infty \\
\stackrel{\infty}{\sigma} \\
\sigma\end{array}$ & 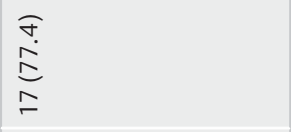 & $\begin{array}{l}\bar{i} \\
+1 \\
\infty \\
\infty \\
\infty\end{array}$ & & 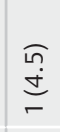 & 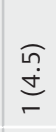 & 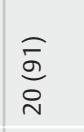 \\
\hline & 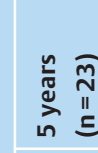 & $\begin{array}{l}\frac{\widehat{p}}{1} \\
\stackrel{n}{N}\end{array}$ & 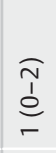 & $\begin{array}{c}\bar{c} \\
e \\
c\end{array}$ & $\begin{array}{l}\bar{I} \\
\frac{1}{c} \\
0\end{array}$ & $\begin{array}{l}\overline{1} \\
\frac{1}{c} \\
0\end{array}$ & 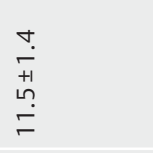 & & $\widehat{\widehat{o}}$ & $\begin{array}{c}\overparen{\ddots} \\
\stackrel{0}{0} \\
\sigma\end{array}$ & 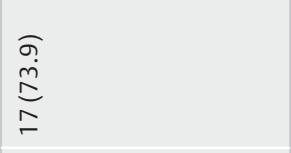 & $\begin{array}{l}\stackrel{\sim}{+} \\
\underset{+1}{+} \\
\dot{\sigma}\end{array}$ & & $\bar{o}$ & $\stackrel{\vec{c}}{\stackrel{m}{c}}$ & $\begin{array}{l}\bar{a} \\
\dot{\infty} \\
\infty \\
\bar{\sim}\end{array}$ \\
\hline & 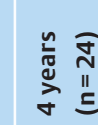 & $\begin{array}{l}\frac{\tilde{p}}{1} \\
\underset{N}{N}\end{array}$ & $\begin{array}{c}\widehat{\hat{T}} \\
\stackrel{d}{\sigma}\end{array}$ & $\begin{array}{c}\overline{1} \\
\stackrel{d}{c}\end{array}$ & $\begin{array}{l}\bar{I} \\
\bar{c} \\
0\end{array}$ & $\begin{array}{l}\overline{1} \\
\bar{o} \\
0\end{array}$ & $\begin{array}{l}\bar{F} \\
+ \\
+1 \\
\stackrel{+}{-} \\
=\end{array}$ & & ô & 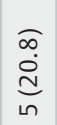 & $\begin{array}{l}\widehat{T} \\
\sigma \\
\Sigma \\
\sigma \\
\sigma\end{array}$ & $\begin{array}{l}\infty \\
\underset{+}{+} \\
+1 \\
\infty\end{array}$ & & ô & $\underset{\substack{m \\
N}}{\infty}$ & $\begin{array}{l}\bar{a} \\
\bar{a} \\
\tilde{N}\end{array}$ \\
\hline & 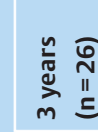 & $\frac{\substack{n \\
\stackrel{1}{n}}}{N}$ & $\frac{\substack{1 \\
d}}{-}$ & $\begin{array}{c}\widehat{T} \\
\stackrel{d}{\sigma} \\
-\end{array}$ & $\begin{array}{l}\bar{T} \\
\stackrel{d}{o} \\
0\end{array}$ & oิ & $\begin{array}{l}\hat{1} \\
\dot{+} \\
\infty \\
\stackrel{+}{+} \\
=\end{array}$ & & $\begin{array}{l}\widehat{\infty} \\
\stackrel{\tilde{c}}{c} \\
-\end{array}$ & $\begin{array}{l}\underset{\sigma}{\stackrel{\rho}{\sigma}} \\
\stackrel{\sigma}{\sigma}\end{array}$ & $\begin{array}{l}\widehat{\alpha} \\
\dot{0} \\
\stackrel{\infty}{\bar{N}} \\
\bar{\sim}\end{array}$ & $\begin{array}{l}\hat{m} \\
+1 \\
\dot{\omega} \\
\dot{\sigma}\end{array}$ & & $\begin{array}{l}\widehat{\infty} \\
\stackrel{0}{c} \\
-\end{array}$ & $\begin{array}{l}\widehat{\infty} \\
\stackrel{n}{c} \\
-\end{array}$ & $\begin{array}{l}\underset{J}{d} \\
\stackrel{d}{\sigma} \\
\stackrel{\sim}{\sim}\end{array}$ \\
\hline & 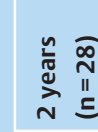 & $\underset{\substack{\hat{1} \\
\sim}}{\stackrel{n}{n}}$ & $\begin{array}{c}\widehat{\hat{\tau}} \\
\stackrel{\underline{\sigma}}{\sigma}\end{array}$ & $\begin{array}{c}\overline{1} \\
\stackrel{c}{c}\end{array}$ & $\begin{array}{c}\overline{1} \\
\stackrel{1}{0}\end{array}$ & 厄) & 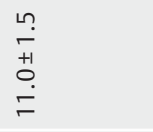 & & $\begin{array}{l}0 \\
\stackrel{0}{0} \\
\stackrel{0}{-}\end{array}$ & $\begin{array}{l}\hat{0} \\
\stackrel{0}{0} \\
-\end{array}$ & $\begin{array}{l}\widehat{a} \\
\infty \\
\infty \\
\stackrel{\infty}{0}\end{array}$ & $\begin{array}{l}\stackrel{9}{+} \\
+1 \\
+1 \\
\infty\end{array}$ & & $\begin{array}{l}\hat{\sigma} \\
\stackrel{0}{0} \\
-\end{array}$ & $\underset{\substack{n \\
n}}{n}$ & $\begin{array}{l}\widehat{a} \\
\infty \\
\stackrel{\infty}{n} \\
\stackrel{n}{N}\end{array}$ \\
\hline & 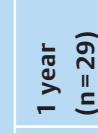 & 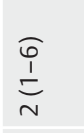 & $\begin{array}{c}\widehat{\hat{d}} \\
\stackrel{d}{e} \\
-\end{array}$ & $\begin{array}{c}\widehat{a} \\
\mathfrak{d} \\
\underline{c}\end{array}$ & $\begin{array}{l}\overline{1} \\
0 \\
0\end{array}$ & $\begin{array}{l}\overline{1} \\
\frac{1}{0} \\
0\end{array}$ & $\begin{array}{l}\stackrel{9}{2} \\
+1 \\
\stackrel{1}{0} \\
0 \\
0\end{array}$ & & $\begin{array}{l}\text { In } \\
\stackrel{n}{c} \\
-\end{array}$ & $\begin{array}{l}\text { గn } \\
\stackrel{n}{n} \\
-\end{array}$ & $\frac{\widehat{N}}{\stackrel{N}{\sim}}$ & $\begin{array}{l}\stackrel{r}{\sim} \\
\stackrel{+}{+} \\
\stackrel{\sim}{\simeq}\end{array}$ & & $\begin{array}{l}\text { గn } \\
\stackrel{n}{c} \\
-\end{array}$ & $\begin{array}{l}\bar{m} \\
\stackrel{0}{c} \\
\stackrel{m}{n}\end{array}$ & $\begin{array}{l}\widehat{\widetilde{v}} \\
\stackrel{\infty}{\infty} \\
\stackrel{\omega}{\sim}\end{array}$ \\
\hline & 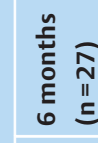 & 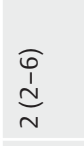 & $\frac{\bar{p}}{d}$ & $\begin{array}{c}\widehat{T} \\
d \\
d \\
-\end{array}$ & $\begin{array}{l}\bar{I} \\
e \\
0\end{array}$ & oิ & $\begin{array}{l}\underset{\sim}{\dot{1}} \\
+1 \\
\stackrel{+}{\leftarrow}\end{array}$ & & $\stackrel{\bar{E}}{\bar{E}}$ & 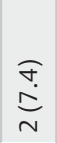 & $\frac{\sqrt[n]{n}}{\stackrel{\infty}{N}}$ & $\begin{array}{l}\stackrel{\sim}{+} \\
+ \\
+1 \\
\infty \\
\stackrel{\sim}{\sim}\end{array}$ & & $\stackrel{\bar{\Xi}}{\bar{\Xi}}$ & 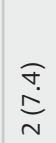 & 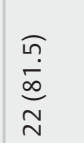 \\
\hline & 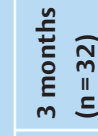 & $\underset{m}{\stackrel{T}{m}}$ & $\underset{\sim}{\tilde{n}}$ & $\begin{array}{l}\widehat{\widehat{T}} \\
\stackrel{d}{v}\end{array}$ & $\begin{array}{l}\overline{1} \\
o \\
0\end{array}$ & $\begin{array}{l}\overline{1} \\
\text { e } \\
0\end{array}$ & $\begin{array}{l}\underset{0}{1} \\
+1 \\
+ \\
\stackrel{\sim}{\longrightarrow}\end{array}$ & & อิ & $\begin{array}{c}\widetilde{0} \\
\stackrel{\infty}{d} \\
\sigma\end{array}$ & $\begin{array}{l}a \\
\stackrel{\sigma}{\vdots} \\
\stackrel{n}{N}\end{array}$ & $\begin{array}{l}0 \\
\stackrel{0}{+} \\
+1 \\
\infty \\
\dot{+} \\
=\end{array}$ & & 1 & 1 & 1 \\
\hline & 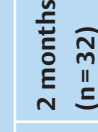 & $\begin{array}{l}\frac{0}{n} \\
\stackrel{n}{m}\end{array}$ & $\begin{array}{l}\widehat{T} \\
\stackrel{d}{d} \\
v\end{array}$ & $\begin{array}{c}\widehat{T} \\
\mathfrak{d} \\
c\end{array}$ & $\begin{array}{l}\bar{T} \\
\frac{1}{0} \\
0\end{array}$ & oิ & 1 & & 1 & 1 & 1 & 1 & & 1 & 1 & 1 \\
\hline 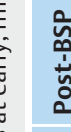 & 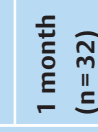 & 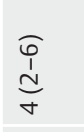 & $\underset{\substack{d \\
d}}{d}$ & $\begin{array}{c}\widehat{I} \\
\stackrel{I}{E} \\
I\end{array}$ & $\begin{array}{c}\widehat{T} \\
\underline{c} \\
c\end{array}$ & ô & 1 & & 1 & 1 & 1 & 1 & & 1 & 1 & 1 \\
\hline & & $\begin{array}{l}\widehat{T} \\
1 \\
e \\
a\end{array}$ & $\frac{\widehat{m}}{\stackrel{n}{n}}$ & $\begin{array}{c}\tilde{m} \\
\mathfrak{d} \\
v\end{array}$ & $\begin{array}{c}\bar{m} \\
\mathfrak{b} \\
-\end{array}$ & $\underset{\substack{1 \\
m}}{\stackrel{n}{d}}$ & $\begin{array}{l}n \\
m \\
+1 \\
\infty \\
\infty \\
\infty\end{array}$ & & $\begin{array}{l}0 \\
\infty \\
\tilde{a} \\
0 \\
0 \\
m\end{array}$ & $\begin{array}{l}\widehat{N} \\
\stackrel{e}{v} \\
N\end{array}$ & $\widehat{\varrho}$ & 1 & & 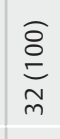 & 1 & 1 \\
\hline & $\begin{array}{l}\widetilde{N} \\
\text { II } \\
\Xi \\
\Xi\end{array}$ & 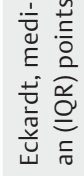 & 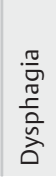 & 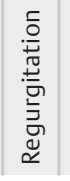 & 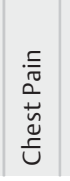 & 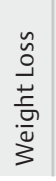 & 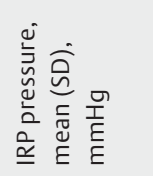 & 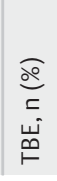 & $\begin{array}{l}\text { ○े } \\
\text { ڤn } \\
\text { v }\end{array}$ & $\begin{array}{l}\text { ळo } \\
\infty \\
0 \\
1 \\
i \\
i n\end{array}$ & $\begin{array}{l}\stackrel{0}{\circ} \\
\stackrel{\infty}{1} \\
\wedge\end{array}$ & 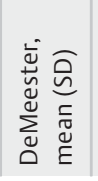 & 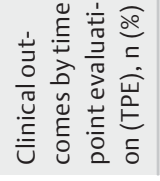 & $\frac{\stackrel{\varrho}{\underline{\Xi}}}{\overline{\bar{\pi}}}$ & $\stackrel{a}{\alpha}$ & ज̆ \\
\hline
\end{tabular}




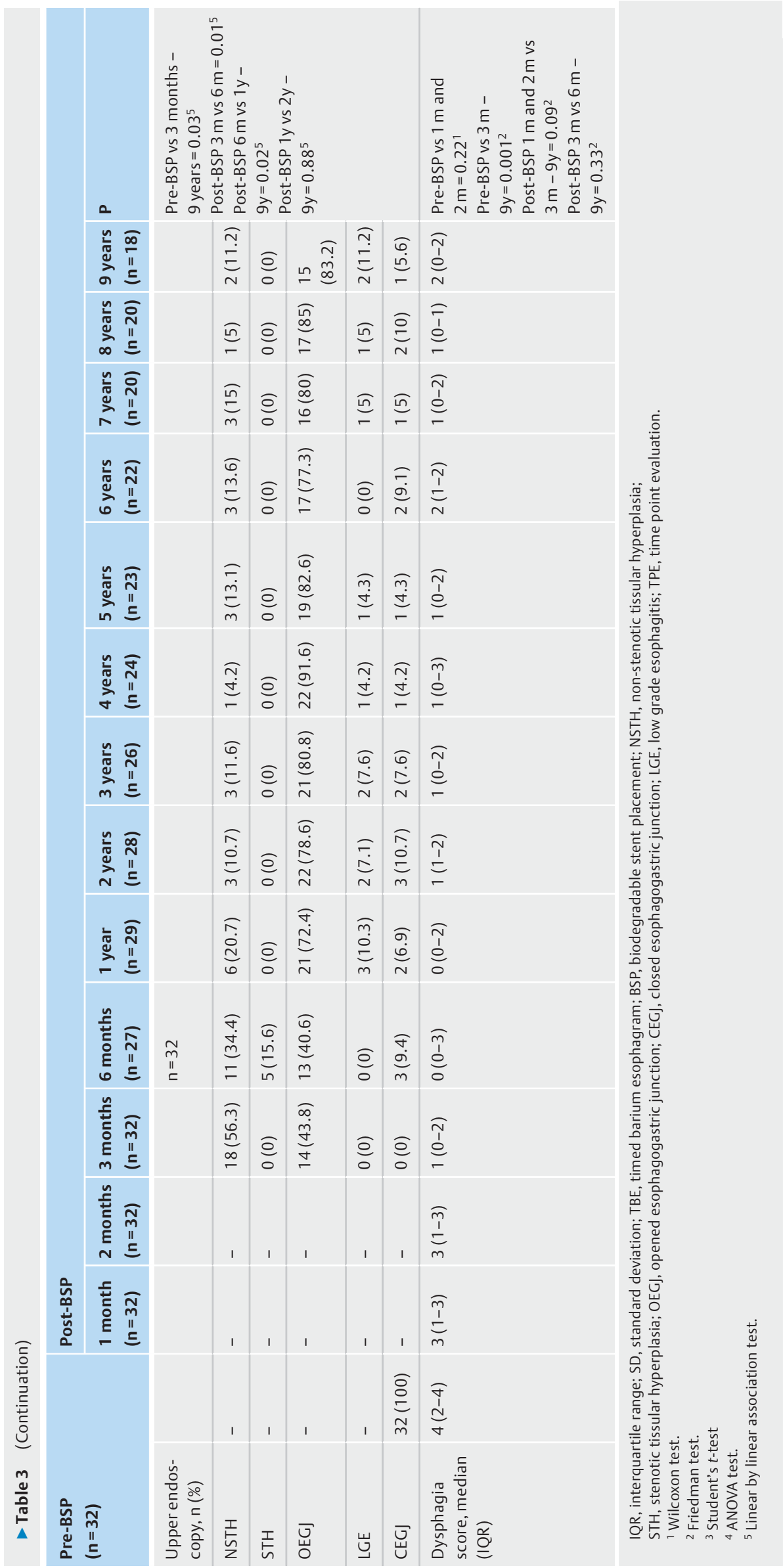




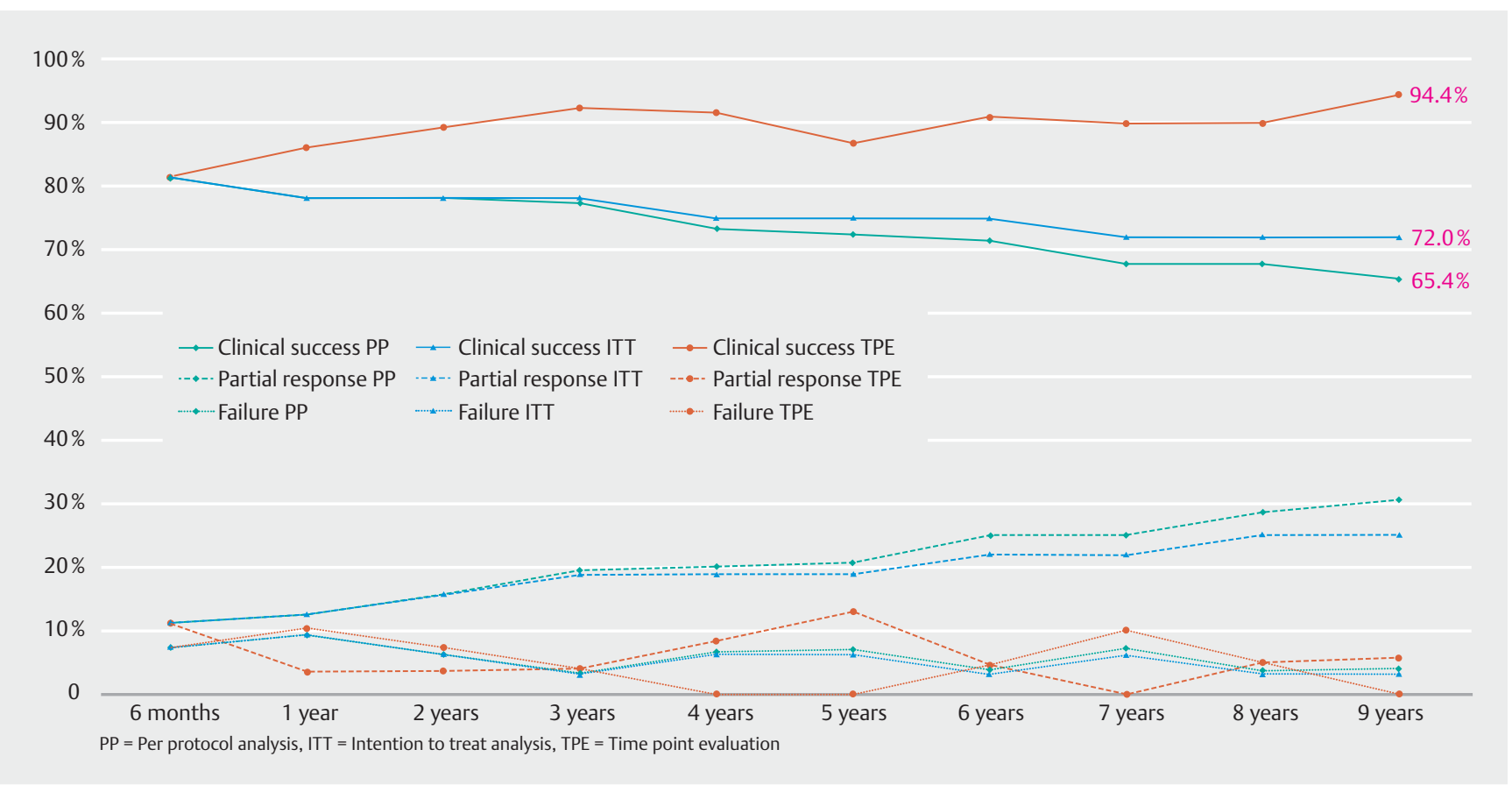

- Fig. 4 BSP outcomes according to follow-up and analysis subtype.

arian patients with achalasia [29-31], with some slight normal differences between populations, but without affecting the results of our study.

Biodegradable stent placement had a technical success of $100 \%$, with a mean placement duration of $37.5 \pm 12.1$ minutes, which is similar to reports using BS in other gastrointestinal diseases (42.1 \pm 14 minutes) $[18,19,22]$. After BSP, our most frequent AE was thoracic pain in 34.4\% (controlled with NSAIDs), similar to other authors $(10 \%-57 \%)[21,22]$, Our migration rate was $(18.7 \%)$ during the first month after BSP, which is similar to other reports $(8 \%-20 \%)[18,20,21]$, and we confirmed that the use of hemoclips in order to avoid this AE could be feasible ( $100 \%$ of efficacy in our cohort, but between $82 \%$ and $95 \%$ in other reports) $[17,18,20,21]$.

Complete degradation was presented as expected in $72.8 \%$ at 3 months, which is similar to the reported mechanism of action of these stents (patency of 6-8 weeks) [17]; at this point, five presented $P R$, of whom three finally presented failure in the next evaluation at 6 months (underwent PD). We think that this time before BS is completely degraded is enough to exert a "continuous dilation" at the EGJ, allowing the clinical effect of these stents in achalasia.

After BSP, we found a direct relationship between clinical dysphagia and endoscopic evaluations at 3- and 6-month assessments. Higher dysphagia scores showed worst endoscopic features. We think that the 6 -month evaluation is the best early TPE in clinical practice, because at this point all stents (including those from second placement) are completely degraded, and clinical effect is available. We observed that patients with scores 2 or 3 at this point have a $60 \%$ probability of performing tissular hyperplasia that will require endoscopy dilation (5 cases in our cohort). Fortunately, response in all cases has an end- point of $15 \mathrm{~mm}$ in less than 5 sessions. This is similar to other reports that show between $30 \%$ and $100 \%$ tissular hyperplasia that requires endoscopic balloon dilation in $40 \%$ to $70 \%$ of cases $[7,8,17,20,21]$.

Clinical success was assessed by TPE, ITT, and PP analysis, based on objective parameters. We confirmed encouraging percentages of $81.5 \%$ at early; $86.9 \%, 76.9 \%$, and $73.4 \%$ at mid-term; and $94.4 \%, 72 \%$, and $64.2 \%$ at long-term evaluation. When success was evaluated at each follow-up, without considering failures or deaths, we had the best percentages (94.4\% at 9 years). However, in the PP and ITT analysis the success decreased to $72 \%$ and $65.4 \%$, respectively, which represents a more realistic clinical evaluation of all the cohort; therefore, we could conclude that BS has long-term clinical efficacy of $65 \%$ to $70 \%$. These results seem to be less effective when compared to other authors. However, SEMS has variable results. Zhao et al [13] reported the surprising clinical success of $100 \%$ at 5 years and $83.3 \%$ at 10 years. This compared to Zeng et al [34], which used FCSEMS and found cumulative clinical remission rates at $6,12,18,24,30$, and 36 months after stent removal of $90.9 \%, 81.8 \%, 76.4 \%, 69.1 \%, 65.5 \%$, and $49.1 \%$, respectively. This could be explained by the lack of complete evaluation methods used. In our case, we tried to include clinical, endoscopic, manometric, radiologic and 24-hour $\mathrm{pH}$ test, coupled with a very strict follow-up, giving us reliable data. POEM is a safe and effective technique with clinical success of $85 \%$ to $100 \%$ at early and mid-term evaluations, but is risky in octogenarians. Abe et al [30] retrospectively compared the feasibility of POEM in a group of octogenarian vs non-octogenarian patients. Octogenarians had a $100 \%$ clinical success at 1 year, but higher incidence of perioperative AE (28.6\% vs $10.2 \%$; $\mathrm{P}$ 0.0001 ), of whom $25 \%$ were major (these included: prolonged 
ICU stay, bleeding/hepatoma requiring blood transfusion, surgery, leak, cardiac arrhythmia and respiratory issues). In our cohort, we had two minor AE: Chest pain in 34\% and intraprocedural bleeding in $12.5 \%$. Sanaka et al [29], compared geriatric (>65 years) vs non-geriatrics (<65 years) retrospectively, and they found no differences in success at 2 months $(94.9 \%$ vs 94.7\%; $\mathrm{P}=\mathrm{NS}$ ), and similar $\mathrm{AE}(10.1 \%$ vs $3.8 \%$; $\mathrm{P}=0.42)$, confirming good safety and efficacy. Chen et al [31], in an international multicenter retrospective study, evaluated 76 octogenarian patients and found at mid-term (265 days), a 93.4\% of clinical success, but up to $14.5 \% \mathrm{AE}$, of whom $7.1 \%$ were severe, confirming, that in spite of POEM being safe and effective in younger patients, serious AE could be presented in octogenarian population, being higher in $\operatorname{LHM}(A E=3 \%-19 \%)$ for these patients [8-10].

Low-grade esophagitis was defined as grade A or B according to LA classification system [35] and it was presented in $4.7 \%$ to $11.2 \%$ of our patients during follow-up. We didn't have more severe cases of reflux disease (grade C, D or Barrett's esophagus). Being these numbers being similar to those found in LHM or even POEM $[5,7,8]$, and all were controlled with PPI medication. DeMeester score was $<14.8$ during follow-up, and we didn't observe any reflux disease complication at long-term, as observed in long-term reports of achalasia when LHM was performed [5, 32].

Our study has some limitations: first, we didn't have a comparison group (with POEM, PD, BTI or MLH), which could have supported our results. Second, we can't extrapolate these results to younger patients, because they were not the objective of our study; however, exploring the effects of BS in those patients as a comparison group could have given us a broader picture of the early, mid and long-term effects of these stents in achalasia at different ages. Third, these results only apply with a 25-mm stent; we didn't explore other diameters, limiting its widespread use; and fourth, this was a single-center study, while a multicenter study could support our data. However, our strengths include: early, mid and long-term evaluations; prospective nature, objective and reproducible evaluations; strict protocol at follow-up, sample size considering the group of patients. Finally, this is the first study that evaluates the role of these stents as an option for achalasia treatment in this population.

\section{Conclusions}

In conclusion, BSP represents a feasible alternative of treatment for octogenarian patients with esophageal achalasia, with acceptable early, mid and long-term results, especially for those who are high-risk for other treatment options. However, comparative and multicenter studies are needed to clarify and confirm the role of these stents in octogenarian patients with achalasia.

\section{Acknowledgements}

The authors thank the Gastroenterology Department staff for the support they provided in development of this protocol.

\section{Competing interests}

The authors declare that they have no conflict of interest.

\section{References}

[1] Swanström L. Achalasia: treatment, current status and future advances. Korean J Intern Med 2019; 34: 1173-1180

[2] Cappell MS, Stravropoulos SM, Friedel D. Updated systematic review of achalasia, with a focus on POEM therapy. Dig Dis Sci 2020; 65: 38 65

[3] Slone S, Kumar A, Jacobs J et al. Accuracy of Achalasia Quality of life and Eckardt scores for assessment of clinical improvement post treatment for achalasia. Dis Esophagus 2020: doi:10.1093/dote/ doaa 080

[4] Vaezi MF, Pandolfino JE, Yadlapati RH et al. ACG Clinical Guidelines: Diagnosis and Management of Achalasia. Am J Gastroenterol 2020; 115: 1393-1411

[5] Fajardo RA, Petrov RV, Bakhos CT et al. Endoscopic and surgical treatments for achalasia: who to treat and how? Gastroenterol Clin North Am 2020; 49: 481-498

[6] Xu S, Chai N, Tang $\mathrm{N}$ et al. Outcomes of peroral endoscopic myotomy in challenging achalasia patients: a long-term follow-up study. Surg Endosc 2020: doi:10.1007/s00464-020-07864-2

[7] Khashab MA, Vela MF, Thosani N et al. ASGE guideline on the management of achalasia. Gastrointest Endosc 2020; 91: 213-227

[8] Oude NijhuisRAB, Zaninotto G, Roman S et al. European Guideline on Achalasia - UEG and ESNM recommendations. United European Gastroenterol J 2020; 8: 13-34

[9] Doubova M, Gowing S, Robaidi H et al. Long-term symptom control following laparoscopic Heller myotomy and Dor fundoplication for achalasia. Ann Thorac Surg 2020: doi:10.1016/j.athoracsur.2020. 06.095

[10] Facciorusso A, Singh S, Fehmi A et al. Comparative efficacy of first-line therapeutic interventions for achalasia: a systematic review and network meta-analysis. Surg Endosc 2020: doi:10.1007/s00464-02007920-x

[11] Van Hoeij F, Tack J, Pandolfino J et al. Complications of botulinum toxin injections for treatment of esophageal motility disorders. Dis Esophagus 2017; 30: 1-5

[12] Cheng YS, Ma F, Li YD et al. Temporary self-expanding metallic stents for achalasia: a prospective study with a long-term follow-up. World J Gastroenterol 2010; 16: 5111-5117

[13] Zhao JG, Li YD, Cheng YS et al. Long-term safety and outcome of a temporary self-expanding metallic stent for achalasia: a prospective study with a 13-year single-center experience. Eur Radiol 2009; 19: 1973-1980

[14] Li TD, Cheng YS, Li MH et al. Temporary self-expanding metallic stents and pneumatic dilation for the treatment of achalasia: a prospective study with long-term follow-up. Dis Esophagus 2010; 23: 361-367

[15] Cai XB, Dai YM, Wan XJ et al. Comparison between botulinum injection and removable covered self-expanding metal stents for the treatment of achalasia. Dig Dis Sci 2013; 58: 1960-1966

[16] Coppola F, Gaia S, Rolle E et al. Temporary endoscopic metallic stent for idiopathic esophageal achalasia. Surg Innov 2014; 21: 11-14

[17] Lorenzo-Zúñiga V, Moreno-de-Vega V, Marin I et al. Biodegradable stents in gastrointestinal endoscopy. World J Gastroenterol 2014; 20: 2212-2217

[18] Hirdes MMC, Siersema PD, Van Boeckel PGA et al. Single and sequential biodegradable stent placement for refractory benign esophageal 
strictures: a prospective follow-up study. Endoscopy 2012; 44: 649654

[19] Dua KS, Vleggaar FP, Santharam R et al. Removable self-expanding plastic esophageal stent as a continuous, non-permanent dilator in treating refractory benign esophageal strictures: a prospective twocenter study. Am J Gastroenterol 2008; 103: 2988-2994

[20] Repici A, Vleggaar FP, Hassan C et al. Efficacy and safety of biodegradable stents for refractory benign esophageal strictures: the BEST (Biodegradable Esophageal Stent) study. Gastrointest Endosc 2010; 72: 927-934

[21] Hair CS, Devonshire DA. Severe hyperplastic tissue stenosis of a novel biodegradable esophageal stent and subsequent successful management with high-pressure balloon dilation. Endoscopy 2010; 42: E132E133

[22] Gkolfakis P, Siersema PD, Tziatzios G et al. Biodegradable esophageal stents for the treatment of refractory benign esophageal strictures. Ann Gastroenterol 2020; 33: 330-337

[23] Bredenoord A], Fox M, Kahrillas P] et al. Chicago classification criteria of esophageal motility disorders defined in high resolution esophageal pressure topography. Neurogastroenterol Motil 2012; 24: 57-65

[24] Kahrilas P], Bredenoord A], Fox M et al. The Chicago Classification of esophageal motility disorders, v3.0. Neurogastroenterol Motil 2015; 27: $160-174$

[25] Eckardt VM, Aignherr C, Bernhard G. Predictors of outcome in patients with achalasia treated by pneumatic dilation. Gastroenterology 1992; 103: 1732-1738

[26] Rezende JM. Classificacao radiológica do megaesófago. Rev Goiana Med 1982; 28: 187-191
[27] Dakkak M, Bennett JR. A new dysphagia score with objective validation. J Clin Gastroenterol 1992; 14: 99-100

[28] Cotton PB, Eisen GM, Aabakken L et al. A lexicon for endoscopic adverse events: report of an ASGE workshop. Gastrointest Endosc 2010; 71: 446-454

[29] Sanaka MR, Chadalavada P, Alomari M et al. Peroral endoscopic myotomy is a safe and effective treatment modality for geriatric patients with achalasia. Esophagus 2020; 17: 484-491

[30] Abe H, Tanaka S, Kawara F et al. Comparison of the safety and efficacy of peroral endoscopic myotomy between octogenarians and non-octogenarians. Dig Endosc 2021; 33: 110-117

[31] Chen $\mathrm{Y}$, Inoue $\mathrm{H}$, Ujiki M et al. An international multicenter study evaluating the clinical efficacy and safety of per-oral endoscopic myotomy in octogenarians. Gastrointest endosc 2018; 87: 956-961

[32] Rosen MJ, Novitsky YW, Cobb WS et al. Laparoscopic Heller myotomy for achalasia in 101 patients: can successful symptomatic outcomes be predicted? Surg Innov 2007; 14: 177-183

[33] Finlayson EV, Birkmeyer JD. Operative mortality with elective surgery in older adults. Eff Clin Pract 2001; 4: 172-177

[34] Zeng Y, Dai YM, Wan X]. Clinical remission following endoscopic placement of retrievable, fully covered metal stents in patients with esophageal achalasia. Dis Esophagus 2014; 27: 103-108

[35] Lundell LR, Dent J, Bennett JR et al. Endoscopic assessment of oesophagitis: clinical and functional correlates and further validation of the Los Angeles Classification. Gut 1999; 45: 172-180 